\title{
Unique Shoulder Arthroscopies: Snapping Scapula Syndrome and Scapular Nerve Entrapment
}

Erica L. Taylor, MS, LAT, ATC, OTC; Spero Karas, MD

Emory Sports Medicine, Atlanta, GA

\section{Full Citation}

Taylor E \& Karas S. Unique shoulder arthroscopies: scapulothoracic bursectomy and spinoglenoid cyst excision. Clin Pract Athl Train. 2020;3(1):6-8. https://doi.org/10.31622/2020/0003.3.

Presented at the $3^{\text {rd }}$ Annual Athletic Trainers in the Physician Practice Society Meeting and Conference, Columbia South Carolina. February 28-29, 2020

\section{ABSTRACT}

Anatomy Review: The scapula provides the stable base for glenohumeral motion and provides origins and insertions for 17 muscles. The scapulothoracic (ST) joint lacks synovial lining and cartilaginous interface. The anterior scapula glides over the thoracic wall between several bursal and soft tissue planes. 1,2 The suprascapular nerve emerges from the superior trunk of the brachial plexus $(\mathrm{C} 5, \mathrm{C} 6)$ and travels across the posterior triangle of the the neck of the scapula trough the suprascapular and spinoglenoid notches. The suprascapular nerve innervates half of the rotator cuff musculature, the infraspinatus and supraspinatus. Snapping Scapula Syndrome (SSS) occurs when the ST articulation impinges on bony or soft tissue structures including a hooking or excessive angulation of the superomedial scapula or bursal inflammation. 1,2,3,4 Patients experience pain with overhead activities accompanied by palpable and/or audible crepitus. Suprascapular Nerve Entrapment (SNE) can occur at the suprascapular notch, affecting both supraspinatus and infraspinatus muscles or at the spinoglenoid notch affecting only the infraspinatus muscle.5,6,7 Extravasation of glenohumeral synovial fluid, due to a torn posterior labrum, can cause a ganglion cyst to appear secondarily. A spinoglenoid cyst causes a patient to have atrophy of the infraspinatus, weakness with external rotation, and posterior shoulder pain or tenderness.

Patient A: The patient was a 30-year-old female. Chief complaint of worsening left posteromedial scapular pain for 6 months with clicking, cracking, and popping of scapula. Recent treatment included 4 months of physical therapy and ST injection by outside MD. Injection provided significant relief and decreased crepitus. Physical exam revealed audible and palpable posterior crepitus with active and passive ROM, tenderness along posteromedial border of scapula. Due to success of previous injection and current worsening symptoms, she consented for diagnostic scapulothoracic arthroscopy. 1,2,3,4 Positioned prone on Wilson frame, max internal rotation/"chicken wing" positioning of arm, surgeon stands opposite surgical side. ${ }^{3,4}$ Intra-operative findings included diffuse scapulothoracic bursitis and prominent superomedial scapular border that were addressed with a bursectomy and recession respectively. At 2 days post-op, she had no palpable/audible crepitus with $90^{\circ}$

AROM/PROM and tenderness along scapula. Physical therapy focused on ROM and scapular stabilization. At 3 months post-op, she had no tenderness long medial scapular border and no recurrence of palpable or audible crepitus.

\section{Discussion Scapulothoracic}

Arthroscopy/Bursectomy: This procedure is indicated when there is pain at the superomedial border of the scapula with painful, audible and/or palpable crepitus that has responded well to injections but failed other conservative measures. Advantages include easy visualization of ST bursa and superomedial border of scapula 
with minimal tissue disturbance. 1,2,3,4 Care should be taken to ensure superficial periosteal layer of superomedial scapula border remains intact. Although long term results are limited, patients have improved outcome scores are 2 years postop with primary and revision procedures. ${ }^{2}$ Injury to the chest well, thoracic cavity, and dorsal scapular nerve and/or artery is possible if portals are not made appropriately $(<3 \mathrm{~m}$ from medial border of scapula). 3,4

Patient B: The patient was a 39-year-old female. Chief complaint of worsening right shoulder pain and weakness for 1 year with insidious onset and failed conservative measures including physical therapy, NSAIDs, and activity modifications. Physical exam revealed marked weakness with external rotation, infraspinatus fossa atrophy and positive special tests including Neers, Hawkins, and External Rotation Lag. MRI revealed posterior labral tear, spinolaminar labral cyst $(2.7 \mathrm{~cm})$ extending into spinoglenoid notch, and edema within infraspinatus. Study was otherwise normal. Due to failed conservative treatment, the patient was consented for arthroscopic posterior Bankart repair and spinoglenoid cyst decompression. 5,6,7 Cyst was decompressed with switching stick/shaver and posterior labral tear (10-6 o'clock) was repaired with Ultra Tape and 4 Micro-Raptor knotless anchors. Given physician's standard Posterior Bankart rehabilitation protocol which included 4 weeks in sling with abduction pillow. At 7 months post-operation, she has regained infraspinatus fossa bulk, significant strength on operative side yet continues to have decreased strength when comparted to uninvolved side. ROM is equal bilaterally.

\section{Discussion Scapulothoracic}

Arthroscopy/Bursectomy: Spinoglenoid notch cysts can be treated in several ways including physical therapy and/or NSAIDs, needle aspiration, labral repair, and cyst excision/decompression +/- labral repair. ${ }^{5}$ When treated operatively, patients can be positioned in beach chair or lateral decubitus. The cyst can be viewed/decompressed in the subacromial or intra-articular spaces. $5,6,7$ In this case, the patient was in lateral decubitus and the cyst was decompressed intra-articularly.

Literature shows no recurrence of surgical decompressed cysts at 6 months post-op $(n=21)$ versus $45 \%$ recurrence with needle aspiration $(n=11) \cdot 5,6$

\section{Correspondence}

Erica Taylor, 10310 The Grove Blvd. Baton Rouge, LA, 70810.

Email: etaylor225@gmail.com

\section{REFERENCES}

1. Menge TJ, Horan MP, Tahal DS, Mitchell JJ, Katthagen JC, Millett PJ. Arthroscopic treatment of snapping scapula syndrome: Outcomes at minimum of 2 Years. Arthroscopy. 2017;33(4):726-732. https://doi.org/10.1016/i.arthro.2016.08.0 29.

2. Tahal DS, Katthagen C, Marchetti DC. A cadaveric model evaluating the influence of bony anatomy and the effectiveness of partial scapulectomy on decompression of the scapulothoracic space in snapping scapula syndrome. AM J Sports Med. 2017;45(6) 1276-1282. https://doi.org $10.1177 / 03635465166877$ 55.

3. Saper M, Kaisk C, Dietzel D. Arthroscopic scapulothoracic decompression for snapping scapula syndrome. Arthrosc Tech. $2015 ; 4(6)$ :e631-636. https://doi.org/10.1016/i.eats.2015.07.00 $\underline{2}$.

4. Karas, Spero. Arthroscopic Resection of the Superior Medial Border of the Scapula. VuMedi.com. www.vumedi.com/video/arthroscopicresection-of-the-superior-medial-border-ofthe-scapula/.

5. Piatt $B$, Hawkins R, Frit, R, Ho C, Wolf E, Schickendantz $M$. Clinical evaluation and 
treatment of spinoglenoid notch ganglion cysts. J Shoulder Elbow Surg.

2002; $11(6): 600-604$.

https://doi.org/10.1067/mse.2002.127094

6. Kim SJ, Choi YR, Jung M, Park JY, Chun YM.

Outcomes of arthroscopic decompression of spinoglenoid cysts through a subacromial approach. Arthroscopy. 2017;33(1):62-67. https://doi.org/10.1016/i.arthro.2016.05.0 34.

7. Ghodadra N, Nho S, Verma, N, Reiff S, Piasecki D, Provencher M, Romeo A. Arthroscopic decompression of the suprascapular nerve at the spinoglenoid notch and suprascapular notch through the subacromial space. Arthroscopy.

2009;25(4):439-445.

https://doi.org/10.1016/i.arthro.2008.10.0 24. 